\title{
Descriptive Analysis of 1972 Cervical Corpectomy Patients and 30-Day Postoperative Outcomes
}

\author{
JAMES E. TOWNER, MD, YAN ICY LI, PHD, THOMAS A. PIETERS, MD, YAN MICHAEL LI, MD, PHD \\ Department of Neurosurgery, University of Rochester Medical Center, Rochester, New York
}

\begin{abstract}
Background: There exists no large, multi-institutional analysis of patients undergoing cervical corpectomy for common degenerative spinal disease, including patient demographics and comorbidities as well as postoperative complications.

Methods: Using Current Procedural Terminology and International Classification of Diseases codes, 1972 patients who underwent a cervical corpectomy for degenerative spinal disease were identified from the American College of Surgeons National Surgical Quality Improvement Program data sets from 2012 to 2015. A descriptive analysis of the patients who underwent the procedure as well as 30-day outcomes and adverse events were collected. Multivariate logistic regression models were used to determine the effect of any preoperative factors identified from a univariate logistic regression analysis (variables with $P<.10$ ) of complications.

Results: The total complication rate, defined as major or minor adverse event, mortality, unplanned readmission, unplanned reoperation, or admission $>30$ days, was $13.28 \%$. The percentage of patients who had $\geq 1$ major or minor adverse events was $5.02 \%$. Several factors commonly associated with an increased risk of perioperative complications, including smoking and diabetes, were found not to be independently associated with complications in this cohort.

Conclusions: This study is the largest analysis of cervical corpectomies. The results of the multivariate analysis provide guidance on risk factors associated with perioperative complications. These data could help develop riskappropriate strategies for minimizing the effects of certain preoperative factors on perioperative complications.
\end{abstract}

Complications

Keywords: cervical corpectomy, vertebrectomy, ACS NSQIP, database, complication, descriptive analysis

\section{INTRODUCTION}

Cervical corpectomies are indicated for pathologies involving the ventral cervical spine, especially when the compressive lesion would not be addressed by completing a discectomy above and below the vertebral body. ${ }^{1}$ Such conditions include degenerative spondylosis, tumors, ossifications of the posterior longitudinal ligament, and infectious processes, among other pathologies. ${ }^{2}$ The largest published series describing cervical corpectomies have been single-institution series of several hundred patients. $^{2-5}$ Although these reviews contain descriptions of risks and adverse events specific to cervical corpectomies, they fail to discuss in any detail the general adverse events associated with undergoing a surgical procedure, nor do they offer in-depth descriptions of patient comorbidities and their association with such adverse events. The purpose of this study is to examine a large, multi-institutional cohort of patients undergoing cervical cor- pectomy for common degenerative spinal conditions. Using data from a large national database, we sought to provide an analysis of the patient population and adverse events among those undergoing cervical corpectomy.

\section{MATERIALS AND METHODS}

\section{Data Source}

The American College of Surgeons National Surgical Quality Improvement Program (ACS NSQIP) is a publicly available, prospectively collected, peer-controlled database created for the examination of 30-day, risk-adjusted surgical outcomes. Database entry is based on a review of patient records via clinically trained personnel. All patient information is de-identified in this nationally available database; thus, no Institutional Review Board evaluation of this study was required. 
Table 1. ICD- 9 codes with $\geq 10$ patients and degenerative pathologies.

\begin{tabular}{|c|c|c|c|}
\hline ICD-9 Code & & Patients & Percentage \\
\hline 721.1 & Cervical spondylosis with myelopathy & 579 & 24.26 \\
\hline 723 & Spinal stenosis in cervical region & 434 & 18.18 \\
\hline 722 & Displacement of cervical intervertebral disc without myelopathy & 271 & 11.35 \\
\hline 721 & Cervical spondylosis without myelopathy & 253 & 10.60 \\
\hline 722.71 & Intervertebral disc disorder with myelopathy, unspecified region & 196 & 8.21 \\
\hline 723.4 & Brachial neuritis or radiculitis NOS & 102 & 4.27 \\
\hline 722.4 & Degeneration of cervical intervertebral disc & 88 & 3.69 \\
\hline 723.1 & Cervicalgia & 30 & 1.26 \\
\hline 722.91 & Other and unspecified disc disorder & 19 & 0.80 \\
\hline Total & & 1972 & 82.61 \\
\hline
\end{tabular}

\section{Patient Identification and Inclusion Criteria}

We examined the ACS NSQIP data set from 2012 to 2015 . Patients ages $\geq 18$ years were identified using Current Procedural Terminology (CPT) codes. We included patients with the following CPT codes: 63081 (vertebral corpectomy, partial or complete, anterior approach with decompression of spinal cord and/or nerve root(s); cervical, single-segment) and 22554 (arthrodesis, anterior interbody technique, including minimal discectomy to prepare interspace [other than for decompression]; cervical below C2). All International Classification of Diseases, 9th revision (ICD-9) diagnosis codes were identified. In an attempt to generate a cohort representative of patients commonly receiving a cervical corpectomy, we excluded groups of ICD-9 diagnosis codes with fewer than 10 patients. Finally, we excluded all nondegenerative diagnoses so as to only include common degenerative conditions in this cohort. Table 1 lists the included ICD-9 diagnoses, as well as the number of patients with each associated diagnosis. The Figure illustrates the patient identification algorithm used to generate our cohort.

\section{Preoperative Description}

Table 2 lists the descriptive variables that were collected. The preoperative comorbidities that were collected are listed in Table 3.

\section{Outcomes Definition}

We examined 6 categories of outcomes: major complications, minor complications, reoperation, an admission lasting $>30$ days, readmission, and 30day mortality. Major and minor complications were tracked from 2012 to 2015 and are listed in Table 4. Beginning in 2013, the NSQIP began tracking mortality, readmissions, and reoperation for the first 30 days after hospital discharge; therefore, data on these outcomes are only for 3 years (2013 through 2015).

\section{Statistical Methods}

Statistical analyses were performed using $\mathrm{R}$ version 3.4.1 (R Foundation for Statistical Computing). Risk factor analysis was completed in a stepwise fashion in order to illuminate true associations and limit the identification of spurious associations. All preoperative factors were first analyzed with a univariate logistic regression model to identify preoperative factors significantly associated with outcomes. Statistical significance was set a priori at an $\alpha$ of 0.10 for the univariate logistic regression model. All variables were noted to have clinical plausibility for being associated with outcomes being investigated, so they were retained for further analysis. Next, these identified variables were subjected to a multivariate logistic regression

\begin{tabular}{|l|l|}
\hline \multicolumn{1}{|c|}{ Patient Identification Algorithm } \\
\hline \multicolumn{1}{|c|}{} \\
\hline$\downarrow$ & NSQIP Database 2012-2015 \\
\hline & Patients >18 years of age with CPT Codes 63081 OR 63081 and 22554 \\
\hline$\downarrow$ & 2387 patients identified \\
\hline & ICD-9 diagnosis with >10 patients (13 codes identified) \\
\hline$\downarrow$ & 2055 patients identified \\
\hline & Excluded patients with ICD-9 diagnoses for non-degenerative conditions (996.5, 730.3, 733.1, \\
\hline & 1972 patients identified \\
\hline
\end{tabular}

Figure. Patient selection algorithm. 
Table 2. Descriptive analysis of cervical corpectomy patient demographics from the NSQIP database.

\begin{tabular}{lrr}
\hline & Number & Percentage \\
\hline Total & 1972 & \\
Sex & & \\
Female & 968 & 49.09 \\
Male & 1004 & 50.91 \\
Race & 1547 & 78.45 \\
White & 8 & 0.41 \\
American Indian or Alaska Native & 34 & 1.72 \\
Asian & 298 & 15.11 \\
Black or African American & 5 & 0.25 \\
Native Hawaiian or Pacific Islander & & \\
Age, y & 166 & 8.42 \\
18-40 & 463 & 23.48 \\
40-50 & 660 & 33.47 \\
$50-60$ & 476 & 24.14 \\
$60-70$ & 185 & 9.38 \\
$70-80$ & 22 & 1.12 \\
>80 & & \\
BMI & 22 & 1.12 \\
18.5 & 379 & 19.22 \\
18.5-24.9 & 673 & 34.13 \\
25-29.9 & 504 & 25.56 \\
30-34.9 & 249 & 12.63 \\
35-39.9 & 145 & 7.35 \\
Smoking status & & \\
No & 1404 & 71.20 \\
Yes & 568 & 28.80 \\
ASAP classification & & \\
1 & 78 & 3.96 \\
2 & 967 & 49.04 \\
3 & 874 & 44.32 \\
3 & 49 & 2.48 \\
Functional health status & & \\
Independent & & 97.21 \\
Partially dependent & 2.18 \\
Totally dependent & 0.25 \\
\hline
\end{tabular}

Abbreviations: ASAP, American Society of Anesthesiology Physical Status Classification; BMI, body mass index.

model to determine which preoperative variables remained statistically significantly associated with our outcome measures of interest. Statistical significance was set a priori at an $\alpha$ of 0.05 for the multivariate logistic regression model.

\section{RESULTS}

Our search identified 1972 adult patients undergoing a cervical corpectomy for common degenerative spinal diagnoses. Tables 2 and 3 include descriptive statistics of the patient characteristics and preoperative factors, respectively. Table 4 lists the rates of adverse events. The total complication rate, defined as major or minor adverse event, mortality, unplanned readmission, unplanned reoperation, or an admission lasting $>30$ days, was $13.28 \%$. The percentage of patients who had $\geq 1$ major or minor adverse events was $5.02 \%$. The most common major adverse event was unplanned reintubation, occurring in $1.12 \%$ of patients. The
Table 3. Descriptive analysis of cervical corpectomy patient comorbidities from the NSQIP database.

\begin{tabular}{lrr}
\hline & Number & Percentage \\
\hline Total & 1972 & \\
Ventilator dependent & 0 & 0.00 \\
History of severe COPD & 90 & 4.56 \\
Ascites & 0 & 0.00 \\
Congestive heart failure in 30 days before & 5 & 0.25 \\
$\quad$ surgery & 968 & 49.09 \\
Hypertension requiring medication & 1 & 0.05 \\
Acute renal failure & 9 & 0.46 \\
Currently on dialysis & 0 & 0.00 \\
Disseminated cancer & 87 & 4.41 \\
Steroid use for chronic condition & 5 & 0.25 \\
>10\% loss of body weight in last 6 mo & & \\
Systemic sepsis & 11 & 0.56 \\
$\quad$ Systemic inflammatory response syndrome & 6 & 0.30 \\
$\quad$ Sepsis & 0 & 0.00 \\
$\quad$ Septic shock & & \\
Diabetes & 139 & 7.05 \\
$\quad$ Insulin dependent & 225 & 11.41 \\
$\quad$ Non-insulin dependent & & \\
Dyspnea & 2 & 0.10 \\
$\quad$ At rest & 105 & 5.32 \\
$\quad$ Moderate exertion & 28 & 1.42 \\
Open wound infection & 5 & 0.25 \\
Bleeding disorders & 9 & 0.46 \\
Preoperative transfusions & & \\
Wound classification & 1948 & 98.78 \\
$\quad$ Clean & 7 & 0.35 \\
Clean/contaminated & 6 & 0.30 \\
Contaminated & 11 & 0.56 \\
Dirty/infected & 17 & 0.86 \\
Emergency case & & \\
\hline Abbreviation COPD, chronic obstructive pulmonary disease. & \\
& &
\end{tabular}

Abbreviation: $\mathrm{COPD}$, chronic obstructive pulmonary disease.

most common minor adverse event was blood transfusion, occurring in $1.57 \%$ of patients.

The 30-day mortality for this group of patients was $0.30 \%$ (6 patients). Four patients had hospitalizations of $>30$ days $(0.20 \%$ of the cohort $)$. The

Table 4. Adverse events reported with cervical corpectomies from the NSQIP database.

\begin{tabular}{lrc}
\hline & Number & Percentage \\
\hline Total & 122 & \\
Major adverse events & & \\
Surgical site infection & 12 & 0.61 \\
Unplanned intubation & 22 & 1.12 \\
Pulmonary embolism & 5 & 0.25 \\
Progressive renal insufficiency & 1 & 0.05 \\
On ventilator $>48$ h & 16 & 0.81 \\
Stroke/CVA & 2 & 0.10 \\
Cardiac arrest requiring CPR & 9 & 0.46 \\
Myocardial infarction & 1 & 0.05 \\
DVT requiring therapy & 9 & 0.46 \\
Sepsis & 14 & 0.71 \\
Septic shock & 3 & 0.15 \\
Minor adverse events & & \\
Wound disruption & 7 & 0.35 \\
Pneumonia & 14 & 0.71 \\
Urinary tract infection & 9 & 0.46 \\
Transfusion & 31 & 1.57 \\
Patients with $\geq 1$ adverse events & 99 & 5.02 \\
\hline
\end{tabular}

Abbreviations: CPR, cardiopulmonary resuscitation; CVA, cerebrovascular accident; DVT, deep venous thrombosis. 
Table 5. Factors associated with complications from multivariate analysis of the NSQIP database.

\begin{tabular}{lcc}
\hline & OR (95\% CI) & $\boldsymbol{P}$ Value \\
\hline Major adverse events & & \\
$\quad$ Severe COPD & $3.97(1.65-8.86)$ & .001 \\
$>10 \%$ loss of body weight & $11.15(1.07-89.14)$ & .027 \\
Emergency case & $9.81(2.51-31.07)$ & $<.001$ \\
Systemic sepsis & $5.91(1.31-21.02)$ & .01 \\
$\quad$ Dependent functional status & $3.41(1.18-8.56)$ & .014 \\
Unplanned readmission & $15.14(3.84-50.66)$ & $<.001$ \\
$\quad$ Wound classification $\geq 2$ & $2.34(1.43-3.92)$ & $<.001$ \\
ASAP classification $\geq 3$ & $8.97(1.09-58.19)$ & .022 \\
Mortality & $12.40(1.88-76.52)$ & .005 \\
$\quad$ Severe COPD & $41.53(0.95-883.38)$ & .031 \\
$\quad$ Steroid use for chronic condition & \\
$>10 \%$ loss of body weight &
\end{tabular}

Abbreviations: ASAP, American Society of Anesthesiology Physical Status Classification; CI, confidence interval; COPD, chronic obstructive pulmonary disease; OR, odds ratio.

rates of unplanned reoperation and unplanned readmission were $2.59 \%$ (51 patients) and $4.01 \%$ (79 patients), respectively.

Table 5 lists the preoperative factors associated with categories of adverse events (major adverse events, readmission, and mortality) from the multivariate analyses. For both mortality and major adverse events, the highest odds ratio (OR) was for patients with $>10 \%$ loss of body weight in the past 6 months, OR $41.534(P=.031)$ and OR $11.153(P=$ $.027)$, respectively. For readmission, the highest OR was associated with any wound classification besides clean $(\mathrm{OR}, 15.138 ; P<.001)$.

\section{DISCUSSION}

Previously published complication rates for cervical corpectomy range from $11 \%$ to $36 \%$, with mortality ranging from 0 to $2.8 \% .^{2-5}$ The mortality rate of $0.3 \%$ in our cohort is on the lower end of the range identified by previously published reports. ${ }^{2-4}$ The total 30-day complication rate, defined as any major or minor adverse events, mortality, unplanned reoperation, unplanned readmission, or an admission lasting $>30$ days, is $13.28 \%$ in our cohort, also lower than previously published singleinstitution, retrospective reviews of cervical corpectomies, which reported rates between $20.8 \%$ and $22.7 \% .^{2,4}$ The categories and subcategories of complications in these previously published reviews included many additional complications that are specific to anterior cervical surgery, such as C5 nerve root palsy, dysphagia, recurrent laryngeal nerve injury, and cerebrospinal fluid leak. However, these previous reports failed to examine many clinically meaningful complications that we incor- porated into our findings. These include the incidence of relatively common issues, such as the need for blood transfusion $(1.57 \%)$ and urinary tract infections $(0.46 \%)$, as well as rarer adverse events that may not occur with enough frequency to be reported in smaller series, like stroke $(0.10 \%)$, renal insufficiency $(0.05 \%)$, and acute renal failure $(0.05 \%)$. Additionally, the indications for surgery in our patient cohort are much more homogenous (i.e., degenerative spinal disease) compared with the prior reports, which include such diverse indications as traumatic injuries, malignancy, and infections. We feel our findings are more applicable to patients with degenerative conditions. ${ }^{3,4}$ It is important to note that our series includes patients who had multilevel cervical corpectomies, which may overestimate the risk profile for single-level corpectomies and may underestimate the risk profile for multilevel cervical corpectomies.

The results of the analysis reveal some interesting associations between preoperative factors and perioperative complications. Perhaps the most surprising finding was that smoking status was not found to be associated with any of the complication categories we assessed, although severe COPD was associated with both major adverse events and 30day mortality on multivariate analysis. Many perioperative complications have been previously associated with smoking, including impaired wound healing, infections, unplanned intubations, and the need for mechanical ventilation. 5,6 However, in a previous study examining smoking status in patients undergoing anterior cervical corpectomy there was only a trend towards an increase in infectious complications in smokers that did not reach statistical significance. ${ }^{5}$ Further, in a prior NSQIP study of posterior cervical spine surgery, smoking was not found to be associated with risk of infection. ${ }^{7}$ We hypothesize that perhaps the smoking cohort included light or occasional smokers who may be at lower risk for perioperative complications compared with heavy smokers, for which having COPD may have functioned as a surrogate marker. Moreover, smoking may not have been found to be associated with infection in this series because the actual incidence of infections was quite low, at $0.61 \%$ (12 of 1972 patients), limiting our ability to statistically determine relationships for this complication in our data set. Previous reports of infection rates range from 0 to $4.3 \% .^{2-4}$ 
Greater than $10 \%$ total body weight loss in the 6 months preceding surgery was found to be associated with major adverse events and mortality. The etiology for the weight loss was not likely due to underlying malignancy, because patients with a diagnosis of malignancy were excluded from this analysis. Therefore, it is a reflection of other underlying comorbidities and/or nutritional deficiency. This potentially modifiable risk factor warrants further study because interventions such as nutrition counseling or diet modification (e.g., high-protein preoperative diet) would be relatively easy to institute.

Data input into ACS NSQIP is done by a trained surgical clinical reviewer at each site with interrater reliability audits conducted at selected sites in order to ensure accurate data entry. ${ }^{8}$ Despite the rigorous steps to ensure valid data, there are limitations to the collected data sets. First, cases are identified based on CPT codes, which introduces coding errors as a cause of misidentifying cases. Because of the large number of operations tracked, the variables must be generic in order to streamline entry. Preventative endeavors, such as perioperative antibiotics or venous thromboembolic prophylaxis, are not recorded, and it is therefore impossible to estimate the effect such measures could have on complication rates. This likely results in an underestimation of the risk of some complications when such preventative efforts are not made. ${ }^{8}$ Also, although there are more than 400 hospitals that contribute data and there is likely less bias than in single-institution studies, the ACS NSQIP data set does not represent a validated nationally representative sample. ${ }^{8}$

As alluded to previously, one major limitation of our analysis is that there are many complications unique to cervical spine surgery that are not captured in the ACS NSQIP database. One of the most common complications is postoperative dysphagia, with rates ranging from $0 \%$ to $58 \% .{ }^{9} \mathrm{C} 5$ nerve root palsy, manifesting as deltoid and/or biceps brachii weakness, is an early postoperative complication, with 2 meta-analyses finding an incidence of $7.5 \%$ for anterior cervical corpectomy and fusion. ${ }^{10,11}$ Other potential complications unique to anterior cervical approaches not reported in the ACS NSQIP data set include perioperative complications, like recurrent laryngeal nerve injury, cerebrospinal fluid leak, and sympathetic chain disruption, as well as long-term morbidity, such as pseudarthrosis rates and adjacent-level stenosis.

Once a condition of the cervical spine has been determined to require operative management, surgical planning must include choosing the approach (anterior, posterior, or combined), the procedure, and how to stabilize the cervical spine, if needed. In the case of a corpectomy, the cervical spine must be reconstructed, which can be completed with an autograft or allograft, with or without additional instrumentation. ${ }^{4}$ Our database study does not provide insight into these details, for which we refer readers to prior studies regarding information on the type of construct used for the fusion, as well as studies comparing cervical corpectomies with other surgical approaches. ${ }^{9,12-19}$

\section{CONCLUSION}

Our study is the largest analysis of cervical corpectomies for common degenerative cervical spinal diseases, adding evidence regarding the general risk profile, including for several adverse outcomes that have not been previously examined. These data are important for the consideration of patient selection and patient counseling prior to surgery. They could additionally help develop riskspecific strategies for minimizing the effects of certain preoperative factors on perioperative complications; for instance, early ambulation or institution of chemical venous thromboprophylaxis for lowering deep venous thrombosis incidence in highrisk patients; aggressive and vigilant wound care; and nutrition modification in high-risk patients. Further development of risk scores or risk calculators could translate these data into a convenient clinical device for guiding decision-making.

\section{REFERENCES}

1. Ghogawala Z. Anterior cervical option to manage degenerative cervical myelopathy. Neurosurg Clin $N \mathrm{Am}$. 2018;29(1):83-89.

2. Ozgen S, Naderi S, Ozek MM, Pamir MN. A retrospective review of cervical corpectomy: indications, complications and outcome. Acta Neurochir (Wien). 2004;146(10):1099-1105; discussion 1105.

3. Mayr MT, Subach BR, Comey CH, Rodts GE, Haid RW, Jr. Cervical spinal stenosis: outcome after anterior corpectomy, allograft reconstruction, and instrumentation. J Neurosurg. 2002;96(1 suppl):10-16.

4. Eleraky MA, Llanos C, Sonntag VK. Cervical corpectomy: report of 185 cases and review of the literature. $J$ Neurosurg. 1999;90(1 suppl):35-41.

5. Lau D, Chou D, Ziewacz JE, Mummaneni PV. The effects 
of smoking on perioperative outcomes and pseudarthrosis following anterior cervical corpectomy: clinical article. $J$ Neurosurg Spine. 2014;21(4):547-558.

6. Turan A, Mascha EJ, Roberman D, Turner PL, You J, Kurz A, et al. Smoking and perioperative outcomes. Anesthesiology. 2011;114(4):837-846.

7. Sebastian A, Huddleston P 3rd, Kakar S, Habermann E, Wagie A, Nassr A. Risk factors for surgical site infection after posterior cervical spine surgery: an analysis of 5,441 patients from the ACS NSQIP 2005-2012. Spine J. 2016;16(4):504-509.

8. User Guide for the 2015 ACS NSQIP Procedure Targeted Participant Use Data File. 2016.

9. Niedzielak TR, Palmer J, Malloy JP 4th. Clinical comparison of surgical constructs for anterior cervical corpectomy and fusion in patients with cervical spondylotic myelopathy or ossified posterior longitudinal ligament: a systematic review and meta-analysis. Clin Spine Surg. 2018;31(6):247-260.

10. Shou F, Li Z, Wang H, Yan C, Liu Q, Xiao C. Prevalence of $\mathrm{C} 5$ nerve root palsy after cervical decompressive surgery: a meta-analysis. Eur Spine J. 2015;24(12):2724-2734.

11. Wang T, Wang H, Liu S, Ding WY. Incidence of C5 nerve root palsy after cervical surgery: a meta-analysis for last decade. Medicine (Baltimore). 2017;96(45):e8560.

12. Kabir SM, Alabi J, Rezajooi K, Casey AT. Anterior cervical corpectomy: review and comparison of results using titanium mesh cages and carbon fibre reinforced polymer cages. Br J Neurosurg. 2010;24(5):542-546.

13. Wen ZQ, Du JY, Ling ZH, Xu HD, Lin XJ. Anterior cervical discectomy and fusion versus anterior cervical corpectomy and fusion in the treatment of multilevel cervical spondylotic myelopathy: systematic review and a meta-analysis. Ther Clin Risk Manag. 2015;11:161-170.

14. Wang T, Wang H, Liu S, An HD, Liu H, Ding WY. Anterior cervical discectomy and fusion versus anterior cervical corpectomy and fusion in multilevel cervical spondylotic myelopathy: a meta-analysis. Medicine (Baltimore). 2016;95(49):e5437.

15. Guan L, Hai Y, Yang JC, Zhou LJ, Chen XL. Anterior cervical discectomy and fusion may be more effective than anterior cervical corpectomy and fusion for the treatment of cervical spondylotic myelopathy. BMC Musculoskelet Disord. 2015;16:29.

16. Han YC, Liu ZQ, Wang SJ, Li LJ, Tan J. Is anterior cervical discectomy and fusion superior to corpectomy and fusion for treatment of multilevel cervical spondylotic myelopathy?: a systemic review and meta-analysis. PLoS One. 2014;9(1):e87191.

17. Huang D, Du X, Liang $\mathrm{H}, \mathrm{Hu} \mathrm{W}, \mathrm{Hu} \mathrm{H}$, Cheng X. Anterior corpectomy versus posterior laminoplasty for the treatment of multilevel cervical myelopathy: a meta-analysis. Int J Surg. 2016;35:21-27.

18. Liu X, Min S, Zhang H, Zhou Z, Wang H, Jin A. Anterior corpectomy versus posterior laminoplasty for multilevel cervical myelopathy: a systematic review and metaanalysis. Eur Spine J. 2014;23(2):362-372.

19. Jiang SD, Jiang LS, Dai LY. Anterior cervical discectomy and fusion versus anterior cervical corpectomy and fusion for multilevel cervical spondylosis: a systematic review. Arch Orthop Trauma Surg. 2012;132(2):155-161.

Disclosures and COI: There are no sources of funding or support for acknowledgment.

Corresponding Author: Yan Michael Li, MD, PhD, Department of Neurosurgery, 601 Elmwood Ave, Rochester, NY 14642. Phone: (585) 2763050; Fax: (585) 756-5183; Email: Yanm_li@ URMC.Rochester.edu.

Published 30 June 2020

This manuscript is generously published free of charge by ISASS, the International Society for the Advancement of Spine Surgery. Copyright @ 2020 ISASS. To see more or order reprints or permissions, see http://ijssurgery.com. 